\title{
Modelos genéricos e específicos para estimativa do volume comercial em uma floresta sob concessão na Amazônia
}

\author{
General and specific models for estimating commercial \\ volume in a forest under concession in Amazon
}

\author{
Vinicius Costa Cysneiros ${ }^{1}$, Allan Libanio Pelissari², Sebastião do Amaral Machado ${ }^{3}$, \\ Afonso Figueiredo Filho ${ }^{4}$ e Luizinho de Souza ${ }^{5}$
}

\begin{abstract}
Resumo
A avaliação da floresta, em termos de seu potencial de madeira comercial, fundamenta tanto as licitações das concessões florestais quanto à autorização de exploração por parte dos órgãos ambientais competentes. Portanto, torna-se necessário o desenvolvimento de novas equações volumétricas com bons níveis de precisão para espécies e formações florestais amazônicas. Este trabalho teve como objetivo ajustar e selecionar modelos para estimativa do volume comercial de espécies em uma floresta sob concessão na Amazônia. Modelos volumétricos foram ajustados para obtenção de uma equação genérica, considerando as 32 espécies comerciais, e de equações específicas para as 12 espécies mais exploradas na área. Com a finalidade de reduzir os erros de estimativa, foram testadas estratificações por classe de diâmetro para todas as equações. Os modelos de Schumacher \& Hall e de Spurr foram estatisticamente os mais adequados, ao passo que as equações específicas e a estratificação por classes de diâmetro proporcionaram maior precisão nas estimativas de volume, sendo recomendadas principalmente para dados oriundos de florestas naturais.
\end{abstract}

Palavras-chave: Floresta tropical, Equações de volume, Espécies madeireiras.

\begin{abstract}
For commercial timber potential, forest evaluation is fundamental for forest concessions and forest operating licensing by environmental agencies. Therefore, it becomes necessary to develop new volumetric equations with good levels of precision for Amazonian forest species. Thus, the aim of this work was to fit and select models to estimate commercial tree species volume in a forest under concession in the Amazonian Forest. Models were fitted to obtain a general equation, considering 32 commercial species; and specific equations for the 12 main species. In order to reduce estimation errors, class diameter stratifications were tested for all equations. The Schumacher \& Hall and Spurr were the best statistical models, whereas specific equations and diameter stratifications provided greater precision for volume estimates, thus being recommended mainly for data from natural forests.
\end{abstract}

Keywords: Tropical Rainforest, Volume equations, Timber species.

\section{INTRODUÇÃO}

A Amazônia Brasileira possui a maior extensão e diversidade biológica entre os remanescentes naturais de floresta tropical (PHILLIPS et al., 2004), com destaque ao mais rico conjunto de espécies vegetais (TER STEEGE et al., 2013). Embora essas florestas apresentem uma infinidade de recursos aproveitáveis, suas riquezas estão dispersas em sua heterogeneidade (CAVALCANTI et al., 2010), em que seus recursos são cada vez mais subaproveitados e desvalorizados.

${ }^{1}$ Doutorando em Engenharia Florestal. UFPR - Universidade Federal do Paraná / Departamento de Ciências Florestais. Av. Prefeito Lothario Meissner - Jardim Botânico - 80210170 - Curitiba, PR, Brasil. E-mail: vccysneiros.florestal@gmail.com

2Professor Adjunto no Departamento de Ciências Florestais. UFPR - Universidade Federal do Paraná. Av. Prefeito Lothario Meissner - Jardim Botânico - 80210170 - Curitiba, PR, Brasil. E-mail: allanpelissari@gmail.com

3Professor Senior no Departamento de Ciências Florestais. UFPR - Universidade Federal do Paraná. Av. Prefeito Lothario Meissner - Jardim Botânico - 80210170 - Curitiba, PR, Brasil. E-mail: samachado@ufpr.br

${ }^{4}$ Professor Associado no Departamento de Engenharia Florestal. UNICENTRO - Universidade Estadual do Centro-Oeste. PR-153 - KM 7 - Bairro Riozinho - 84500-000 - Irati, PR, Brasil. E-mail: afigfilho@gmail.com

${ }^{5}$ Coordenador de Operações Florestais. Amata Brasil S/A. Estrada da Balsa, Km 1, Setor Industrial - $76861-000$ - Itapuã do Oeste, RO, Brasil. E-mail: luizinho.souza@amatabrasil.com.br 
Por muito tempo, a quantificação volumétrica das espécies amazônicas foi efetuada com base no fator de forma de 0,7 proposto por Heinsdijk e Bastos (1963). Esse valor foi empregado de forma generalizada para diferentes espécies, sítios, formações e tipologias florestais, ocasionando sérios erros nas estimativas volumétricas (HIRAMATSU, 2008; ROLIM et al., 2006). No início da década de 1980, aflorou a necessidade pelo aprimoramento das técnicas para estimar o volume de árvores, visando à obtenção de estimativas mais confiáveis. Desde então, diversos trabalhos foram realizados na Amazônia com esse objetivo (COLPINI et al., 2009; HIGUCHI; RAMM, 1985; LEITE; RESENDE, 2010; GIMENEZ et al., 2015; ROLIM et al., 2006; TONINI; BORGES, 2015).

Na Floresta Amazônica, a abundância de espécies florestais das mais variadas dimensões afeta, de sobremaneira, a forma das árvores, conferindo diversas peculiaridades, o que dificulta, por consequência, o emprego de fatores de forma e equações de volume (FIGUEIREDO FILHO, 1983). A heterogeneidade pronunciada na composição de espécies, até mesmo dentro de uma pequena área, constitui um importante desafio no desenvolvimento de funções de volume para florestas tropicais naturais, em que o ajuste de equações específicas é uma das principais formas de contornar essa dificuldade (AKINDELE; LEMAY, 2006).

No contexto das concessões florestais no Brasil, o desenvolvimento de novas equações de volume para espécies e formações florestais amazônicas é necessário para subsidiar as ações de planejamento e ordenamento florestal. Diversas etapas do manejo em regime de concessão requerem estimativas precisas do volume comercial das árvores, desde a avaliação da floresta para o embasamento das licitações, até a emissão da Autorização de Exploração por parte dos órgãos ambientais competentes, justificando assim o aprimoramento dessas equações.

Com base no exposto, este trabalho teve como objetivo ajustar e selecionar modelos volumétricos genéricos e específicos para as principais espécies comerciais de uma floresta em concessão na Amazônia, avaliando estratégias de estratificação por classes diamétricas e de agrupamentos de equações por identidade de modelos.

\section{MATERIAL E MÉTODOS}

\section{Área de estudo}

A área de estudo está localizada na Floresta Nacional do Jamari, a qual abrange os municípios de Candeias do Jamari, Itapuã do Oeste e Cujubim, no Norte do estado de Rondônia, entre as coordenadas geográficas $09^{\circ} 00^{\prime} 00^{\prime \prime}$ a $09^{\circ} 30^{\prime} 00^{\prime \prime} \mathrm{S}$ e $62^{\circ} 44^{\prime} 05^{\prime \prime}$ a $63^{\circ} 16^{\prime} 64^{\prime \prime} \mathrm{W}$. Ocupando uma área de aproximadamente 220.000 ha, a Flona do Jamari apresenta relevo plano a suavemente ondulado em quase toda sua extensão e clima Tropical Chuvoso do tipo Aw (Köppen), com período seco bem definido na estação do inverno, precipitação média anual em torno de $2.400 \mathrm{~mm}$ e temperatura média anual de $25^{\circ} \mathrm{C}$.

$\mathrm{Na}$ área predominam as tipologias de Floresta Ombrófila, variando de Aberta à Densa. A Floresta Ombrófila Densa é caracterizada pelo domínio de fanerófitos nas subformas de vida macro e mesofanerófitos, com destaque para as arbóreas de grande porte: Dinizia excelsea Ducke, Bertholletia excelsea Humb. \& Bonpl., Cariniana micrantha Ducke e Huberodedron switenioides (Gleason) Ducke (IBGE, 2012). Nas formações abertas, predominam palmeiras, como Attalea speciosa Mart. ex. Spreng. e Oenocarpus bataua Mart., e bambus do gênero Guadua, com destaque da Musaceae: Phenakospermum guyannense (A. Rich.) Endl. ex Miq.

\section{Análise dos dados}

Os dados utilizados para o ajuste dos modelos volumétricos foram provenientes da cubagem em romaneio das árvores exploradas entre os anos de 2014 e 2015, gerando um banco de dados com 5.231 árvores e 15.750 toras, distribuídas em 32 espécies (Tabela 1). O método de Smalian foi adotado para a cubagem, onde na base e no topo de cada tora foram medidos os diâmetros no menor e no maior raio, obtendo-se o valor médio. A cubagem foi efetuada para a determinação do volume $(v)$ na altura comercial das árvores $(h)$, em que os diâmetros a 1,3 m do solo $(d)$ foram obtidos dos inventários florestais efetuados nas Unidades de Produção, por meio de placas de identificação e códigos específicos fixados nos indivíduos arbóreos com diâmetro igual ou superior a $40 \mathrm{~cm}$. 
Tabela 1. Estatísticas descritivas das variáveis utilizadas nos modelos volumétricos para as espécies amazônicas.

Table 1. Descriptive statistics of the variables used in volumetric models for Amazonian species.

\begin{tabular}{|c|c|c|c|c|c|c|c|c|c|c|}
\hline \multirow{2}{*}{ Espécie } & \multirow{2}{*}{ Amostras } & \multicolumn{3}{|c|}{ d } & \multicolumn{3}{|c|}{$\mathbf{h}$} & \multicolumn{3}{|c|}{$\mathbf{v}$} \\
\hline & & minimo & médio & máximo & minimo & médio & máximo & minimo & médio & máximo \\
\hline Allantoma decandra Ducke & 70 & 50.0 & 80.5 & 140.0 & 13.5 & 21.7 & 31.8 & 2.49 & 7.14 & 19.85 \\
\hline Apuleia leiocarpa (Vogel) J.F.Macbr. & 152 & 57.0 & 87.7 & 165.0 & 10.6 & 20.1 & 31.2 & 2.43 & 8.47 & 24.42 \\
\hline Astronium lecointei Ducke & 767 & 50.3 & 75.6 & 146.4 & 7.3 & 26.7 & 43.4 & 1.60 & 7.72 & 28.75 \\
\hline Bagassa guianensis Aubl. & 14 & 57.0 & 84.6 & 124.0 & 16.8 & 19.6 & 24.0 & 2.55 & 7.12 & 15.10 \\
\hline Bowdichia nitida Spruce ex Benth. & 28 & 50.3 & 64.6 & 89.0 & 13.6 & 20.7 & 26.9 & 2.81 & 4.98 & 9.03 \\
\hline Brosimum rubescens Taub. & 127 & 51.0 & 75.9 & 134.0 & 9.4 & 17.8 & 23.5 & 2.29 & 5.53 & 12.18 \\
\hline Cariniana micrantha Ducke & 123 & 62.0 & 114.4 & 188.0 & 16.8 & 22.8 & 29.2 & 3.18 & 16.59 & 37.62 \\
\hline Caryocar glabrum Pers. & 143 & 51.3 & 79.9 & 150.0 & 6.1 & 14.6 & 21.9 & 2.28 & 5.87 & 18.98 \\
\hline Caryocar villosum (Aubl.) Pers. & 69 & 57.3 & 90.4 & 143.0 & 6.2 & 15.2 & 23.5 & 1.85 & 7.37 & 19.41 \\
\hline Cedrela fissilis Vell. & 14 & 52.5 & 75.0 & 99.0 & 10.5 & 15.4 & 19.5 & 1.89 & 4.08 & 9.44 \\
\hline Cedrelinga cateniformis (Ducke) & 57 & 57.0 & 97.7 & 205.0 & 5.2 & 19.4 & 29.8 & 2.15 & 10.17 & 29.60 \\
\hline Clarisia racemosa Ruíz \& Pav. & 292 & 51.0 & 70.5 & 105.7 & 5.2 & 15.7 & 25.2 & 1.44 & 4.43 & 10.22 \\
\hline Cordia goeldiana Huber & 24 & 57.0 & 77.5 & 105.0 & 20.6 & 25.7 & 36.1 & 3.84 & 7.21 & 15.74 \\
\hline Couratari stellata A. C. Sm. & 506 & 60.0 & 88.3 & 210.0 & 13.9 & 25.8 & 39.9 & 2.52 & 10.64 & 35.25 \\
\hline Dinizia excelsa Ducke & 555 & 55.7 & 106.1 & 245.0 & 8.0 & 19.2 & 34.2 & 2.62 & 14.65 & 55.74 \\
\hline Diplotropis rodriguesii H.C. Lima & 14 & 54.4 & 66.1 & 80.9 & 12.7 & 20.5 & 27.5 & 3.06 & 4.30 & 6.96 \\
\hline Dipteryx alata Vogel & 7 & 52.0 & 66.3 & 76.0 & 15.1 & 18.2 & 21.8 & 2.10 & 5.83 & 7.89 \\
\hline Dipteryx odorata (Aubl.) Willd. & 289 & 50.3 & 75.6 & 207.0 & 7.8 & 17.0 & 31.3 & 1.73 & 5.57 & 18.13 \\
\hline Erisma bicolor Ducke & 103 & 53.0 & 80.7 & 150.0 & 11.4 & 19.3 & 28.9 & 2.37 & 6.47 & 21.72 \\
\hline Erisma fuscum Ducke & 87 & 51.0 & 74.9 & 134.0 & 10.0 & 20.2 & 28.9 & 2.31 & 5.87 & 15.88 \\
\hline Goupia glabra Aubl. & 184 & 53.0 & 83.2 & 143.2 & 5.4 & 14.7 & 29.7 & 1.80 & 6.00 & 22.24 \\
\hline Handroanthus impetiginosus (Mart. ex DC.) Mattos & 16 & 67.0 & 90.0 & 117.8 & 20.8 & 26.4 & 32.2 & 4.63 & 12.36 & 21.98 \\
\hline Handroanthus incanus (A.H. Gentry) S. O. Grose & 62 & 52.5 & 75.2 & 114.9 & 13.8 & 27.0 & 34.3 & 2.56 & 6.70 & 22.37 \\
\hline Hymenaea intermedia Ducke & 77 & 52.0 & 72.3 & 132.0 & 13.4 & 21.4 & 30.0 & 2.19 & 6.32 & 17.54 \\
\hline Hymenolobium heterocarpum Ducke & 323 & 52.0 & 95.2 & 216.0 & 7.6 & 20.5 & 36.2 & 1.90 & 11.06 & 49.23 \\
\hline Manilkara elata (Allemão ex Miq.) Monach. & 13 & 50.9 & 70.3 & 103.1 & 18.1 & 21.9 & 25.8 & 2.66 & 5.23 & 8.25 \\
\hline Mezilaurus synandra (Mez) Kosterm. & 31 & 60.5 & 73.9 & 102.5 & 7.7 & 17.3 & 26.7 & 1.84 & 5.05 & 11.19 \\
\hline Peltogyne paniculata Benth. & 740 & 50.0 & 68.3 & 178.0 & 7.8 & 16.5 & 33.9 & 1.41 & 4.11 & 10.80 \\
\hline Peltogyne venosa (Vahl) Benth. & 10 & 57.0 & 77.7 & 114.6 & 11.9 & 18.8 & 24.7 & 1.88 & 5.26 & 9.14 \\
\hline Pouteria guianensis Aubl. & 65 & 50.3 & 64.6 & 104.1 & 11.9 & 18.4 & 28.4 & 2.04 & 3.96 & 7.99 \\
\hline Qualea paraensis Ducke & 244 & 50.0 & 67.9 & 119.0 & 10.4 & 22.4 & 40.7 & 1.88 & 5.37 & 13.87 \\
\hline Simarouba amara Aubl. & 23 & 50.3 & 60.4 & 78.0 & 11.6 & 19.3 & 26.2 & 2.03 & 3.57 & 7.78 \\
\hline
\end{tabular}

Em que: $\mathrm{d}$ = diâmetro a l,3 m do solo; $\mathrm{h}=$ altura comercial; $\mathrm{v}=$ volume comercial sem casca; mínimo = valor mínimo; médio = valor médio; e máximo $=$ valor máximo.

Devido à extensão e variabilidade dos dados, a etapa de pré-processamento foi aplicada por espécie para detecção de outliers. A identificação desses valores discrepantes foi realizada com base no teste de Grubbs (GRUBBS, 1969). De forma complementar, foi realizada a análise gráfica da dispersão da variável dependente volume $(v)$ pela variável independente diâmetro a 1,3 $\mathrm{m}$ do solo (d), sendo excluídos os pares de observação com baixa correlação e isoladas da nuvem de dispersão.

Os outliers são dados com comportamento distinto aos demais, podendo representar erros não amostrais ou eventos discrepantes de uma população (GRUBBS, 1969), sendo uma fonte potencial de problemas em ajustes de equações preditivas. Para a detecção desses dados, foram selecionados apenas os valores extremos do teste de Grubbs $\left(G>2 . G_{\text {tabelado }}\right)$ e os pares de variáveis de baixa correlação, que se encontraram isoladas e distantes da nuvem de dispersão, evitando a remoção das árvores de grande porte características da Floresta Amazônica.

Para a construção das equações de volume, 11 modelos volumétricos (Tabela 2) contidos na literatura florestal (CLUTTER et al., 1983; LOETSCH et al., 1973) foram ajustados por meio de regressão linear. $\mathrm{O}$ ajuste desses modelos foi realizado para obtenção de uma equação genérica, considerando todas as 32 espécies cubadas, e de equações específicas para as 12 principais espécies exploradas, resultando em treze ajustes para cada modelo. 
Tabela 2. Modelos volumétricos ajustados para estimativa do volume comercial das espécies amazônicas.

Table 2. Volumetric models fitted to estimate the commercial volume of Amazonian species.

\begin{tabular}{llll}
\hline Modelo & Autor & Modelo matemático & Variáveis de entrada \\
\hline 1 & Berkhant & $v=\beta_{0}+\beta_{1} \cdot d+\varepsilon i$ & \\
2 & Kopezky \& Gehrhardt & $v=\beta_{0}+\beta_{1} \cdot d^{2}+\varepsilon \mathrm{i}$ & Simples \\
3 & Hohenald \& Krenn & $v=\beta_{0}+\beta_{1} \cdot d+\beta_{2} \cdot d^{2}+\varepsilon i$ & \\
4 & Husch & $\ln v=\beta_{0}+\beta_{1} \cdot \ln d+\varepsilon i$ & \\
5 & Brenac & $\ln v=\beta_{0}+\beta_{1} \cdot \ln d+\beta_{2} \cdot 1 / d+\varepsilon \mathrm{i}$ & \\
\hline 6 & Spurr & $v=\beta_{0}+\beta_{1} \cdot\left(\mathrm{d}^{2} \cdot h\right)+\varepsilon \mathrm{i}$ & \\
7 & Spurr Logarítimica & $\ln v=\beta_{0}+\beta_{1} \cdot \ln \left(d^{2} \cdot h\right)+\varepsilon \mathrm{i}$ & Dupla \\
8 & Schumacher \& Hall & $\ln v=\beta_{0}+\beta_{1} \cdot \ln d+\beta_{2} \cdot \ln h+\varepsilon \mathrm{i}$ & \\
9 & Stoate & $v=\beta_{0}+\beta_{1} \cdot d^{2}+\beta_{2} \cdot\left(d^{2} \cdot h\right)+\beta 3 \cdot h+\varepsilon \mathrm{i}$ & \\
10 & Meyer & $v=\beta_{0}+\beta_{1} \cdot d+\beta_{2} \cdot d^{2}+\beta 3 \cdot(d \cdot h)+\beta 4 \cdot\left(d^{2} \cdot h\right)+\beta 5 \cdot h+\varepsilon i$ & \\
11 & Naslund & $v=\beta_{0}+\beta_{1} \cdot d^{2}+\beta_{2} \cdot\left(d^{2} \cdot h\right)+\beta 3 \cdot\left(d \cdot h^{2}\right)+\beta 4 \cdot h^{2}+\varepsilon i$ & \\
\hline
\end{tabular}

Em que: $v=$ volume comercial sem casca $\left(\mathrm{m}^{3}\right) ; \mathrm{d}=$ diâmetro a altura do peito $(\mathrm{cm})$, medido a I,3 $\mathrm{m}$ do solo; $\mathrm{h}=$ altura comercial $(\mathrm{m}) ; \mathrm{ln}=$ logaritmo neperiano; e $\varepsilon i=$ erro associado.

A seleção dos melhores modelos foi baseada no maior coeficiente de determinação ajustado ( $\mathrm{R}^{2} \mathrm{aj}$.) e no menor erro padrão da estimativa em porcentagem (Syx\%), os quais foram recalculados para os modelos logarítmicos, em que a variável dependente sofreu alterações. De forma complementar, a análise gráfica dos resíduos foi realizada para avaliar tendências nas estimativas, bem como a homogeneidade das variâncias dos modelos, em que a distribuição aleatória e sem tendência dos erros foi um indicativo de homoscedasticidade.

O erro sistemático gerado nos modelos logarítmicos foi corrigido pelo fator de Meyer (FCM) e a significância dos coeficientes avaliada a partir do p-valor $\leq 0,01$, visando à identificação de possíveis problemas com multicolinearidade. Para validação dos melhores modelos por meio do teste de aderência do Qui-quadrado $\left(\chi^{2}\right)$, foram selecionadas amostras independentes de $10 \%$ dos dados para equação genérica e de 15\% para as equações específicas.

Com a finalidade de reduzir os erros de estimativa, estratificações por classe de diâmetro, com intervalos de 20, 30 e $50 \mathrm{~cm}$, foram testadas para todas as equações. Para verificar a possibilidade de agrupamento das equações, foi aplicado o teste de identidade proposto por Graybill, o qual consiste na redução da soma dos quadrados para verificar, pelo teste F, a significância da diferença de um modelo completo para o reduzido (QUEIROZ et al., 2008).

\section{RESULTADOS E DISCUSSÃO}

\section{Análise de outliers}

Assim, foi observada a manutenção das medidas de tendência $(\bar{y})$ e de variabilidade $\left(\sigma, \sigma^{2}\right.$, CV\% e $\lambda$ ) após a remoção dos outliers, com pouca variação na densidade do banco de dados $(\mathrm{N})$ e com aumento nas correlações (r) (Tabela 3).

Tabela 3. Estatísticas descritivas da população com outliers e após refinamento sem outliers.

Table 3. Descriptive statistics of the population with outliers and after refinement without outliers.

\begin{tabular}{|c|c|c|c|c|c|c|c|c|c|}
\hline \multirow{2}{*}{ Tratamento } & \multirow{2}{*}{$\overline{\mathbf{y}}$} & \multirow{2}{*}{$\sigma$} & \multirow{2}{*}{$\sigma^{2}$} & \multirow{2}{*}{ CV\% } & \multirow{2}{*}{$\lambda$} & \multirow{2}{*}{$\mathbf{N}$} & \multicolumn{3}{|c|}{$r$} \\
\hline & & & & & & & $d \times v$ & $d \times h$ & $h \times v$ \\
\hline Com outliers & 7,90 & 5,70 & 32,49 & 72,19 & 54,33 & 5231 & 0,7856 & 0,1235 & 0,3476 \\
\hline Sem outliers & 7,82 & 5,37 & 28,93 & 68,74 & 36,44 & 5150 & 0,8021 & 0,1248 & 0,3594 \\
\hline
\end{tabular}

Em que: $\bar{y}=$ média dos volumes reais em $\mathrm{m}^{3} ; \sigma=$ desvio padrão; $\sigma^{2}=$ variância; $\mathrm{CV} \%=$ coeficiente de variação; $\lambda=$ amplitude do volume real em $\mathrm{m}^{3} ; \mathrm{N}=$ número de árvores cubadas; $\mathrm{r}=$ coeficiente de correlação linear de Pearson; $\mathrm{d}=$ diâmetro à altura do peito; $\mathrm{h}=$ altura comercial; $\mathrm{e} v=$ volume real.

\section{Ajuste e seleção dos modelos}

Os modelos de dupla entrada foram estatisticamente superiores aos de simples entrada (Tabela 4), com os menores valores de erro padrão da estimativa (Syx\%) e os maiores coeficientes de determinação ajustado ( $\left.R^{2} a j\right)$. A melhora significativa dos ajustes, com a inserção da variável altura, é citada por diversos autores, como Barreto et al. (2014); Rolim et al. (2006), Thaines et al. (2010), e Tonini e Borges (2015). No entanto, a estratificação vertical das florestas tropicais dificulta a mensuração dessa variável, prejudicando, assim, a estimativa do volume (SEGURA; KANNINEN, 2005), sendo recomendada cautela em sua mensuração e inserção nos modelos. 
Tabela 4. Coeficientes estimados e estatísticas de ajuste dos melhores modelos volumétricos de simples e dupla entrada selecionados para as equações genéricas e específicas.

Table 4. Estimated coefficients and fit statistics of best single and dual input volumetric models selected for general and specific equations.

\begin{tabular}{|c|c|c|c|c|c|c|c|c|c|}
\hline Equação & Modelo & $\beta_{0}$ & $\beta_{1}$ & $\beta_{2}$ & Syx\% & $R^{2}$ aj. & FCM & $\chi^{2}$ calculado & $\chi^{2}$ tabelado \\
\hline \multirow{2}{*}{ Genérica } & 1 & $-8,096$ & 0,195 & - & 41,09 & 0,625 & - & 458,22 & \multirow{2}{*}{467,17} \\
\hline & 8 & $-8,273$ & 1,804 & 0,763 & 35,97 & 0,713 & 1,036 & 433,21 & \\
\hline \multirow{2}{*}{ Apuleia leiocarpa } & 1 & $-5,736$ & 0,163 & - & 35,16 & 0,524 & - & - & \multirow{2}{*}{28,96} \\
\hline & 7 & $-7,674$ & 0,815 & - & 32,20 & 0,601 & 1,042 & 7,82 & \\
\hline \multirow{2}{*}{ Astronium lecointei } & 1 & $-6,094$ & 0,184 & - & 27,72 & 0,599 & - & - & \multirow{2}{*}{90,35} \\
\hline & 8 & $-8,210$ & 1,662 & 0,922 & 24,60 & 0,684 & 1,021 & 41,14 & \\
\hline \multirow{2}{*}{ Brosimum rubescens } & 1 & $-3,686$ & 0,122 & - & 29,10 & 0,487 & - & - & \multirow{2}{*}{9.39} \\
\hline & 8 & $-7,658$ & 1,525 & 0,948 & 24,32 & 0,642 & 1,026 & 7,46 & \\
\hline \multirow{2}{*}{ Caryocar glabrum } & 2 & 1,378 & 0,001 & - & 31,39 & 0,559 & - & - & \multirow{2}{*}{10,85} \\
\hline & 6 & 1,951 & 0,000 & - & 27,62 & 0,659 & - & 15,53 & \\
\hline \multirow{2}{*}{ Clarisia racemosa } & 1 & $-2,418$ & 0,097 & - & 23,30 & 0,431 & - & - & \multirow{2}{*}{28,96} \\
\hline & 6 & 1,043 & 0,000 & - & 17,54 & 0,678 & - & 7,80 & \\
\hline \multirow{2}{*}{ Couratari stellata } & 1 & $-6,761$ & 0,200 & - & 31,48 & 0,531 & - & - & \multirow{2}{*}{56,92} \\
\hline & 8 & $-7,955$ & 1,656 & 0,880 & 28,77 & 0,609 & 1,033 & 60,26 & \\
\hline \multirow{2}{*}{ Dinizia excelsea } & 3 & $-14,868$ & 0,368 & $-0,001$ & 37,64 & 0,448 & - & - & \multirow{2}{*}{60,39} \\
\hline & 7 & $-6,758$ & 0,763 & - & 35,94 & 0,496 & 1,057 & 152,68 & \\
\hline \multirow{2}{*}{ Dipteryx odorata } & 4 & $-6,355$ & 1,855 & - & 31,09 & 0,639 & 1,042 & - & \multirow{2}{*}{28,96} \\
\hline & 7 & $-7,798$ & 0,825 & - & 27,52 & 0,717 & 1,029 & 17,07 & \\
\hline \multirow{2}{*}{ Goupia glabra } & 4 & $-5,857$ & 1,712 & - & 31,89 & 0,582 & 1,043 & - & \multirow{2}{*}{16,93} \\
\hline & 8 & $-7,295$ & 1,599 & 0,726 & 28,84 & 0,658 & 1,030 & 15,76 & \\
\hline \multirow{2}{*}{$\begin{array}{l}\text { Hymenolobium } \\
\text { heterocarpum }\end{array}$} & 1 & $-8,490$ & 0,208 & - & 35,24 & 0,630 & - & - & \multirow{2}{*}{32,27} \\
\hline & 8 & $-8,092$ & 1,644 & 0,975 & 31,82 & 0,698 & 1,036 & 95,73 & \\
\hline \multirow{2}{*}{$\begin{array}{l}\text { Peltogyne } \\
\text { paniculatum }\end{array}$} & 4 & $-3,480$ & 1,151 & - & 28,93 & 0,268 & 1,041 & - & \multirow{2}{*}{86,79} \\
\hline & 8 & $-5,303$ & 1,093 & 0,742 & 25,12 & 0,448 & 1,029 & 31,24 & \\
\hline \multirow{2}{*}{ Qualea paraensis } & 2 & 0,723 & 0,001 & - & 25,77 & 0,577 & - & - & \multirow{2}{*}{22,47} \\
\hline & 6 & 0,932 & 0,000 & - & 22,02 & 0,691 & - & 9,14 & \\
\hline
\end{tabular}

Em que: $\beta_{0}, \beta_{1}$ e $\beta_{2}=$ coeficientes da regressão a serem estimados; Syx $\%=$ erro padrão da estimativa em porcentagem; $\mathrm{R}^{2}$ aj. $=$ coeficiente de determinação ajustado; e FCM = fator de correção de Meyer; $\chi_{\text {calculado }}^{2}=$ valor Qui-quadrado calculado para validação dos modelos selecionados; e $\chi_{\text {tabelado }}^{2}=$ valor Qui-quadrado tabelado para validação dos modelos selecionados ao nível de probabilidade de $95 \%$.

De maneira geral, os modelos de simples entrada de Hohenald \& Krenn e de Brenac e os de dupla entrada de Stoat, Meyer e Naslund apresentaram problema de multicolinearidade. Neste estudo, optou-se por excluir os modelos que apresentaram coeficientes de regressão não significativos ( $p$-valor $\leq 0,01)$. Assim, os modelos de Schumacher \& Hall (8) e o de Spurr (6 e 7) resultaram nas estimativas mais precisas do volume comercial. Esses modelos são frequentemente citados como os mais adequados para a Floresta Amazônica, tanto para equações genéricas (COLPINI et al., 2009; ROLIM et al., 2006; THAINES et al., 2010; TONINI; BORGES, 2015) quanto para específicas (MOURA, 1994; RIBEIRO et al., 2014).

A análise gráfica dos resíduos para as equações genéricas (Figura 1) evidenciou menor tendência de superestimativa para o modelo de Spurr Logarítmico (7), em comparação ao modelo de Schumacher \& Hall (8) selecionado. Para o modelo de Spurr (7), a variável $d^{2}$ oferece maior peso e, devido sua alta correlação com o volume $(\mathrm{r}=0,802)$, possibilitou minimizar as superestimativas. Essa análise deve ser determinante na seleção do melhor modelo, mesmo que os demais critérios indiquem outro (DRAPER; SMITH, 1998). Logo, o modelo de Spurr Logaritmo (7) foi o mais adequado para a composição de uma equação genérica para a estimativa do volume comercial $(d<$ $50 \mathrm{~cm}$ ) na Floresta Nacional do Jamari. A análise gráfica também foi decisiva para a seleção das melhores equações específicas (Figura 2), por meio da identificação de tendências, principalmente de heterocedasticidade.

Essa análise reforçou a elevada variabilidade das variáveis dendrométricas das espécies amazônicas, uma vez que os maiores resíduos estiveram diretamente relacionados às espécies de grande porte (Figura 2), conforme mencionado por Brandeis et al. (2006) para florestas tropicais; como exemplo de D. excelsea e A. lecointei no presente estudo. Cabe ressaltar que a utilização de toras de grande comprimento, oriundas de romaneio, não compromete o ajuste de modelos volumétricos, conforme constatado por Ribeiro et al. (2014), ao avaliar diferentes seccionamentos para cubagem e escolha de equações na Floresta Nacional do Tapajós, estado do Pará. 
(A) Modelo 7 - Spurr

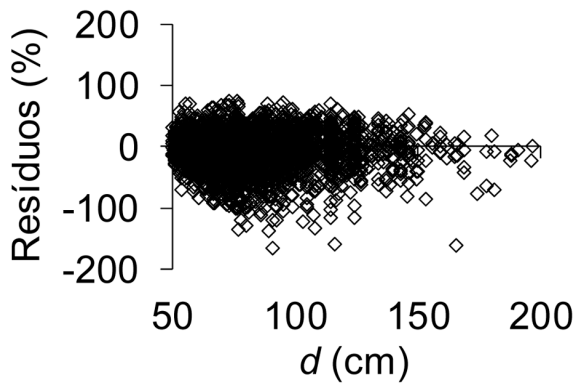

(B) Modelo 8 - Schumacher \& Hall

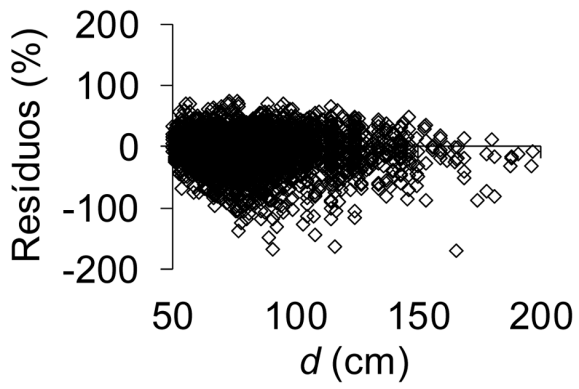

Figura 1. Distribuição dos resíduos para os melhores modelos genéricos na estimativa do volume comercial. Figure 1. Residuals distributions for best general models selected to estimate commercial volume.

(A) Apuleia leiocarpa

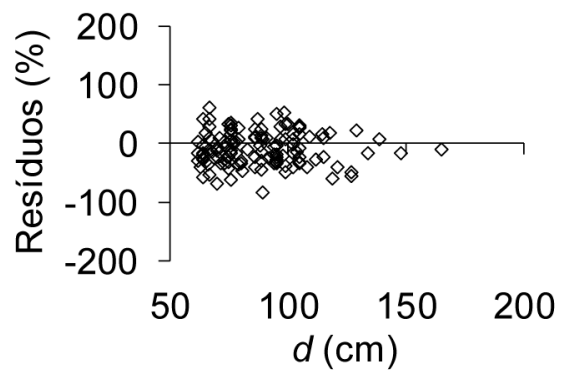

(D) Caryocar glabrum

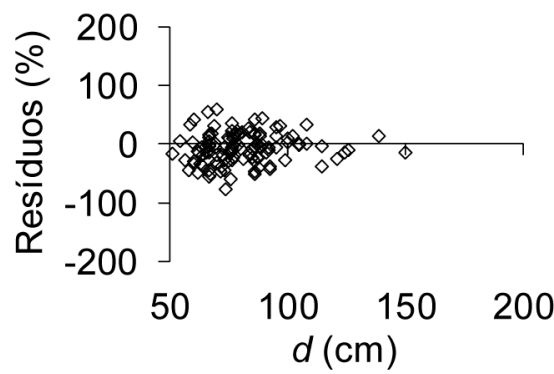

(G) Dinizia excelsea

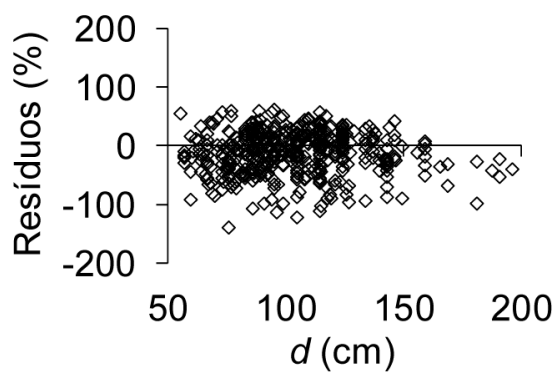

(J) Hymenolobium heterocarpum

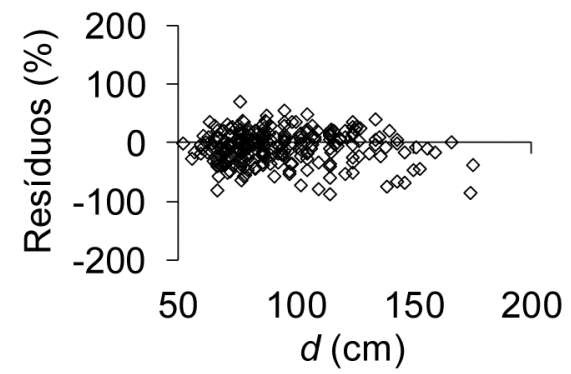

(B) Astronium lecointei

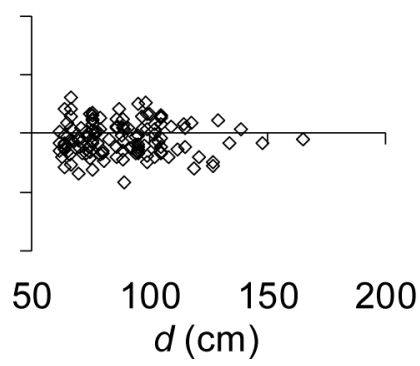

(E) Clarisia racemosa

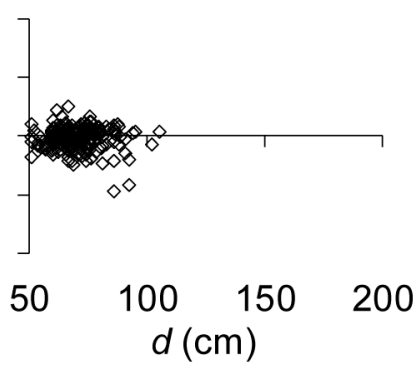

(H) Dipteryx odorata

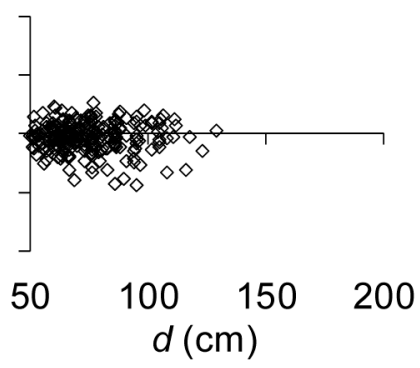

(L) Peltogyne paniculatum

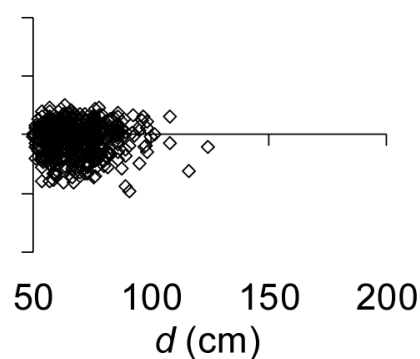

(C) Brosimum rubescens

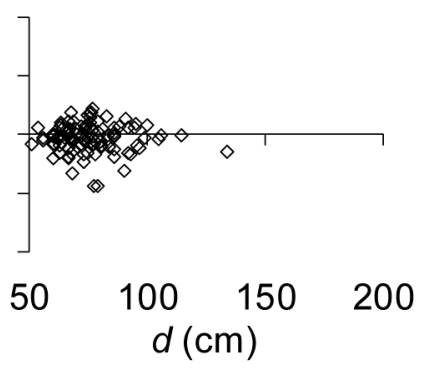

(F) Couratari stellata

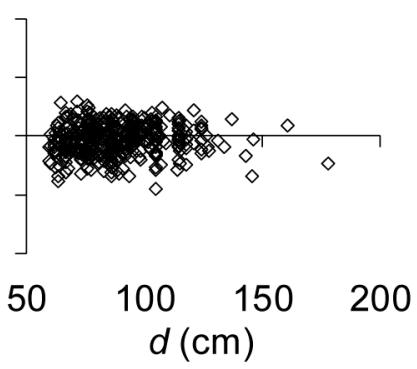

(I) Goupia glabra

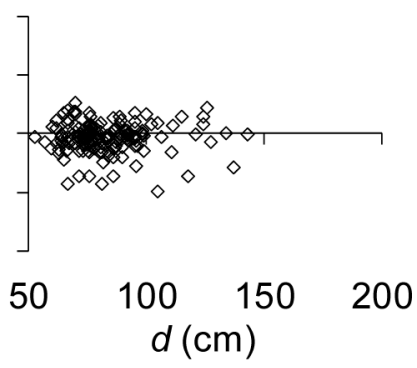

(M) Qualea paraensis

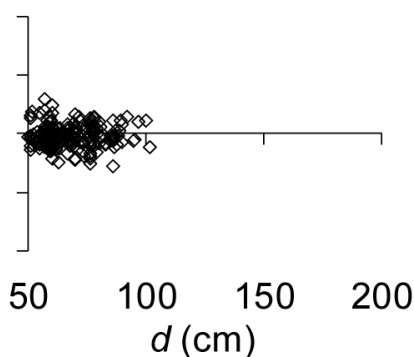

Figura 2. Distribuição dos resíduos para os melhores modelos específicos na estimativa do volume comercial de espécies amazônicas.

Figure 2. Residuals distribution for the best specific models to estimate commercial volume of Amazonian species. 
Os dois melhores modelos selecionados para a equação genérica foram validados pelo teste do $\chi^{2}$ ao nível de probabilidade de $95 \%$ (Tabela 4 ). O valor de $\chi_{\text {calculado }}^{2}$ foi menor que o $\chi_{\text {tabelado }}^{2}$ aceitando-se a hipótese que os modelos selecionados são aderentes ao conjunto de dados. Para as equações específicas de dupla entrada selecionadas, todas foram validadas pelo teste do $\chi^{2}$, com exceção de $H$. heterocarpum, C. stellata, D. excelsea e C. glabrum, sendo essas as espécies de maior porte e responsáveis pelos maiores erros nos ajustes.

\section{Estratificação}

Em geral, as últimas classes diamétricas apresentaram baixa frequência de observações e em número insuficiente para o emprego consistente da regressão linear. Nesses casos, as classes anteriores tiveram seus limites superiores abertos, tornando a estratificação mais flexível, o que acarretou na redução de 1 a 14\% do erro padrão da estimativa para a equação genérica, considerando as classes diamétricas em relação a toda amostra (Tabela 5).

Tabela 5. Estratificações diamétricas recomendadas para as melhores equações genéricas e específicas, com os respectivos coeficientes de regressão e estatísticas de ajuste.

Table 5. Diameter stratifications indicated for general and specific equations, with their regression coefficients and fit statistics.

\begin{tabular}{|c|c|c|c|c|c|c|c|}
\hline Equação & Modelo & Classe de diâmetro (cm) & $\beta_{0}$ & $\beta_{1}$ & $\beta_{2}$ & Syx\% & $\mathbf{R}^{2} \mathbf{a j}$ \\
\hline \multirow{7}{*}{ Genérica } & \multirow{7}{*}{8} & $50<70$ & $-6,600$ & 1,452 & 0,690 & 25,98 & 0,420 \\
\hline & & $70<90$ & $-8,965$ & 1,917 & 0,824 & 32,88 & 0,404 \\
\hline & & $90<110$ & $-11,416$ & 2,451 & 0,832 & 32,17 & 0,344 \\
\hline & & $110<130$ & $-8,594$ & 1,981 & 0,596 & 34,01 & 0,172 \\
\hline & & $130<150$ & $-4,709$ & 1,167 & 0,616 & 29,45 & 0,146 \\
\hline & & $150<170$ & 4,358 & $-0,547$ & 0,517 & 28,46 & 0,011 \\
\hline & & $170<$ & $-21,122$ & 4,102 & 0,973 & 22,01 & 0,287 \\
\hline \multirow{3}{*}{ Apuleia leiocarpa } & \multirow{3}{*}{7} & $50<80$ & $-9,648$ & 0,988 & - & 35,98 & 0,257 \\
\hline & & $80<110$ & $-7,750$ & 0,823 & - & 31,94 & 0,240 \\
\hline & & $110<$ & $-8,935$ & 0,909 & - & 24,75 & 0,529 \\
\hline \multirow{4}{*}{ Astronium lecointei } & \multirow{4}{*}{8} & $50<70$ & $-7,348$ & 1,425 & 0,953 & 21,79 & 0,442 \\
\hline & & $70<90$ & $-8,735$ & 1,748 & 0,971 & 22,66 & 0,387 \\
\hline & & $90<110$ & $-11,295$ & 2,479 & 0,732 & 19,64 & 0,463 \\
\hline & & $110<$ & $-11,718$ & 2,536 & 0,666 & 33,59 & 0,212 \\
\hline \multirow{3}{*}{ Brosimum rubescens } & \multirow{3}{*}{8} & $50<70$ & $-7,046$ & 1,553 & 0,681 & 21,60 & 0,337 \\
\hline & & $70<90$ & $-6,751$ & 1,328 & 0,941 & 25,45 & 0,260 \\
\hline & & $90<$ & $-7,217$ & 1,138 & 1,400 & 20,05 & 0,586 \\
\hline \multirow{2}{*}{ Caryocar glabrum } & \multirow{2}{*}{6} & $50<100$ & 1,406 & 0,000 & - & 30,46 & 0,401 \\
\hline & & $100<150$ & 3,109 & 0,000 & - & 16,66 & 0,653 \\
\hline \multirow{2}{*}{ Clarisia racemosa } & \multirow{2}{*}{6} & $50<70$ & 1,132 & 0,000 & - & 18,15 & 0,475 \\
\hline & & $70<$ & 1,010 & 0,000 & - & 16,86 & 0,576 \\
\hline \multirow{2}{*}{ Couratari stellata } & \multirow{2}{*}{8} & $50<100$ & $-8,446$ & 1,785 & 0,857 & 27,87 & 0,468 \\
\hline & & $100<$ & $-5,412$ & 1,118 & 0,879 & 26,81 & 0,259 \\
\hline \multirow{3}{*}{ Dinizia excelsea } & \multirow{3}{*}{7} & $50<100$ & $-7,497$ & 0,825 & - & 41,22 & 0,316 \\
\hline & & $100<150$ & $-5,360$ & 0,654 & - & 31,38 & 0,261 \\
\hline & & $150<200$ & $-4,886$ & 0,606 & - & 20,87 & 0,261 \\
\hline \multirow{3}{*}{ Dipteryx odorata } & \multirow{3}{*}{8} & $50<80$ & $-8,197$ & 1,728 & 0,851 & 26,76 & 0,514 \\
\hline & & $80<110$ & $-8,773$ & 1,913 & 0,748 & 26,33 & 0,444 \\
\hline & & $110<140$ & $-9,865$ & 2,404 & 0,304 & 23,30 & 0,131 \\
\hline \multirow{3}{*}{ Goupia glabra } & \multirow{3}{*}{8} & $50<80$ & $-7,740$ & 1,696 & 0,746 & 26,33 & 0,364 \\
\hline & & $80<110$ & $-10,276$ & 2,239 & 0,749 & 22,81 & 0,485 \\
\hline & & $110<140$ & $-5,476$ & 1,531 & 0,205 & 35,02 & $-0,205$ \\
\hline \multirow{3}{*}{$\begin{array}{l}\text { Hymenolobium } \\
\text { heterocarpum }\end{array}$} & \multirow{3}{*}{8} & $50<100$ & $-8,917$ & 1,860 & 0,934 & 33,06 & 0,472 \\
\hline & & $100<150$ & $-6,601$ & 1,348 & 0,957 & 27,24 & 0,297 \\
\hline & & $150<200$ & $-8,729$ & 1,214 & 1,801 & 17,48 & 0,608 \\
\hline \multirow{3}{*}{ Peltogyne paniculatum } & \multirow{3}{*}{8} & $50<70$ & $-5,568$ & 1,132 & 0,781 & 24,46 & 0,365 \\
\hline & & $70<90$ & $-6,090$ & 1,274 & 0,740 & 24,68 & 0,300 \\
\hline & & $90<$ & $-2,343$ & 1,157 & $-0,432$ & 22,97 & $-0,007$ \\
\hline \multirow{2}{*}{ Qualea paraensis } & & $50<80$ & $-6,986$ & 1,549 & 0,667 & 23,05 & 0,532 \\
\hline & 8 & $80<110$ & $-10,490$ & 2,234 & 0,824 & 19,15 & 0,424 \\
\hline
\end{tabular}

Em que: $\beta_{0}$, $\beta_{1}$ e $\beta_{2}=$ coeficientes da regressão a serem estimados; Syx\% = erro padrão da estimativa em porcentagem; e $\mathrm{R}^{2}$ aj. $=$ coeficiente de determinação ajustado. 
Essa estratificação proporcionou redução da variância dentro dos estratos, tornando-os mais homogêneos quanto a variável de interesse. Assim, os melhores resultados foram obtidos pela estratificação em classe de $20 \mathrm{~cm}$ de diâmetro. No entanto, os menores intervalos requereram maior número de equações, cabendo ao gestor florestal decidir se a melhora na precisão das estimativas não será inviabilizada pelo maior tempo de processamento. Quanto às equações específicas, a influência da estratificação foi bastante diferenciada, variando de suave redução até aumento do Syx\%. No entanto, a análise dos resíduos indicou aumento na precisão das estimativas, principalmente com a redução das superestimativas.

Ao empregar a estratificação em modelos de regressão, bons resultados são citados para relações hipsométricas (DAVID et al., 2014) e para modelos de afilamento (SOUZA et al., 2012). Contudo, essa técnica ainda é pouco explorada para modelos volumétricos em floresta tropicais, sobretudo na Floresta Amazônica, onde diversos fatores operacionais e do ambiente dificultam a obtenção de estimativas consistentes.

\section{Identidade dos modelos}

As possibilidades de agrupamento das equações foram avaliadas por meio do teste de identidade de Graybill, o qual testa a hipótese das equações serem estatisticamente semelhantes e da viabilidade de utilizar apenas um único modelo para o conjunto de dados. Assim, ao comparar a equação genérica com as específicas (Tabela 6 ), o teste foi não significativo $\left(\mathrm{F}_{\text {calculado }}<\mathrm{F}_{\text {tabelado }}\right.$ ) ao nível de confiança 99\%, indicando que não há diferença entre as equações avaliadas.

Essas combinações de equações, juntamente com suas respectivas estratificações recomendadas, foram empregadas para avaliar os modelos por estrato de classes de diâmetro. Com isso, obteve-se resultado não significativo para a equação genérica, ao passo que o teste indicou significância apenas para Clarisia racemosa e Caryocar glabrum nas equações específicas, resultando na necessidade de estratificação.

Tabela 6. Análise de variância do teste de Graybill para os modelos gerais e reduzidos.

Table 6. Analysis of variance of the Graybill's test for the general and reduced models.

\begin{tabular}{lccccc}
\hline Fontes de variação & GL & SQ & QM & F & F tab (1\%) \\
\hline Modelo completo & 36 & 61336 & - & - & - \\
Modelo reduzido & 3 & 60241 & - & - & - \\
Redução & 33 & 1096 & 33,20 & $0,463 \mathrm{~ns}$ & 1,664 \\
Resíduo & 4594 & 329369 & 71,70 & - & - \\
Total & 4630 & 390705 & - & - & - \\
\hline
\end{tabular}

Em que: $\mathrm{GL}=$ graus de liberdade; $\mathrm{SQ}=$ soma de quadrados; $\mathrm{QM}=$ quadrado médio; $\mathrm{F}=$ teste $\mathrm{F} ; \mathrm{F}$ tab $=$ valor de $\mathrm{F}$ tabelado; e $\mathrm{ns}=$ não significância ao nível de confiança de $99 \%$.

\section{CONCLUSÕES}

Os modelos de Schumacher e Hall (8) e de Spurr ( 6 e 7) proporcionam os melhores ajustes na composição de equações genéricas e específicas para as espécies comerciais em área de manejo sob concessão na Floresta Amazônica. Os modelos de simples entrada apresentam resultados satisfatórios e podem ser recomendados para as estimativas de volume. Ao passo que as equações específicas e o emprego da estratificação por classes de diâmetro proporcionam elevação da precisão das estimativas de volume, sobretudo pela redução da variabilidade dos dados.

\section{REFERÊNCIAS BIBLIOGRÁFICAS}

AKINDELE, S. O.; LEMAY, V. M. Development of tree volume equations for common timber species in the tropical rain forest area of Nigeria. Forest Ecology and Management, Amsterdam, v. 226, p. 41-48, 2006.

BARRETO, W. F.; LEÃO, F. M.; MENEZES, M. C.; SOUZA, D. V. Equação de volume para apoio ao manejo comunitário de empreendimento florestal em Anapu, Pará. Pesquisa Florestal Brasileira, Brasília, v. 34, n. 80, p. 321-329, 2014. 
BRANDEIS, T. J.; DELANEY, M.; PARRESOL, B. R.; ROYER, L. Development of equations for predicting Puerto Rican subtropical dry forest biomass and volume. Forest Ecology and Management, Amsterdam, v. 233, n. 1, p. 133-142, 2006.

CAVALCANTI, F. J. B.; MACHADO, S. A.; HOSOKAWA, R. T. Metodologia para uso múltiplo e integrado de florestas tropicais na Amazônia. Floresta, Curitiba, v. 40, n. 2, p. 405-418, 2010.

CLUTTER, J.; FORTSON, J. C.; PIENAAR, L. V.; BRISTER, G. H.; BAILEY, R. L. Timber management: a quantitative approach. New York: John Wiley e Sons, 1983. 333 p.

COLPINI, C.; TRAVAGIN, D. P.; SOARES, T. S.; SILVA, V. S. M. Determinação do volume, do fator de forma e da porcentagem de casca de árvores individuais em uma Floresta Ombrófila Aberta na região noroeste de Mato Grosso. Acta Amazonica, Manaus, v. 39, n. 1, p. 97-104, 2009.

DAVID, H. C.; MARINHESKI FIlHO, A.; PEliSSARI, A. L.; PÉlliCO NETTO, S.; ARAúJO, E. J. G.; BAUM, L. Critérios de estratificação para o ajuste de funções de afilamento em fustes de pinus. Pesquisa Florestal Brasileira, Brasília, v. 34, n. 79, p. 197-206, 2014.

DRAPER, N. R.; SMITH, H. Applied regression analysis. 3 ed. New York: John Wiley e Sons, 1998.704 p.

FIGUEIREDO FILHO, A. Estudos de modelos matemáticos para estimar o volume por unidade de área em uma floresta tropical úmida na Amazônia brasileira. 1983. 176 p. Dissertação (Mestrado em Ciências) - Universidade Federal do Paraná, Curitiba, 1983.

GIMENEZ, B. O.; DANIELLI, F. E.; OLIVEIRA, C. K. A.; SANTOS, J.; HIGUCHI, N. Equações volumétricas para espécies comerciais madeireiras do sul do estado de Roraima. Scientia Forestalis, Piracicaba, v. 43, n. 106, p. 291-301, 2015.

GRUBBS, F. E. Procedures for Detecting Outlying Observations in Samples. Technometrics, v. 11, n. 1, p. 1314, 1969.

HEINSDIJK, D.; BASTOS, A. M. Inventários florestais na Amazônia. Boletim do Serviço Florestal, Brasília, v. 6, p. 1-100, 1963.

HIGUCHI, N.; RAMM, W. Developing bole wood volume equations for a group of tree species of central Amazon (Brazil). The Commonwealth Forestry Review, v. 64, n. 1, p. 33-41, 1985.

HIRAMATSU, N. A. Equações de volume comercial para espécies nativas na região do Vale do Jari, Amazônia Oriental. 2008. 107 p. Dissertação (Mestrado em Engenharia Florestal) - Universidade Federal do Paraná, Curitiba, 2008.

IBGE - INSTITUTO BRASILEIRO DE GEOGRAFIA E ESTATÍSTICA. Manual Técnico da Vegetação Brasileira. 2 ed.. Rio de Janeiro: IBGE, 2012. 271 p.

LEITE, F. S.; RESENDE, A. V. Estimativa do volume de madeira partindo do diâmetro da cepa em uma área explorada de floresta amazônica de terra firme. Ciência Florestal, Santa Maria, v. 20, n. 1, p. 69-79, 2010.

LOETSCH, F.; ZÖHRER, F; HALLER, K. E. Forest inventory. Munich: BLV, 1973. 469 p.

MOURA, J. B. Estudo de forma do fuste e comparação de métodos de estimativa volumétrica de espécies florestais da Amazônia Brasileira. 1994. 114 p. Dissertação (Mestrado em Engenharia Florestal) - Universidade Federal do Paraná, Curitiba, 1994. 
Cysneiros et al. - Modelos genéricos e específicos para estimativa do

volume comercial em uma floresta sob concessão na Amazônia

PHILliPS, P. D.; AZEVEDO, C. P.; DEGEN, B.; THOMPSON, I. S.; SILVA, J. N. M.; VAN GARDINGEN, P. R. An individual based spatially explicit simulation model for strategic forest management planning in the eastern Amazon. Ecological Modelling, Kidlington, v. 173, p. 335-354, 2004.

QUEIROZ, D.; MACHADO, S. A.; FIGUEIREDO FILHO, A.; ARCE, J. E.; KOEHLER, H. S. Identidade de modelos em funções de afilamento para Mimosa scabrella Bentham em povoamentos nativos da região metropolitana de Curitiba/PR. Floresta, Curitiba, v. 38, n. 2, p. 339-349, 2008.

RIBEIRO, R. B. S.; GAMA, J. R. V.; MELO, L. O. Seccionamento para cubagem e escolha de equações de volume para a Floresta Nacional do Tapajós. Cerne, Lavras, v. 20, n. 4, p. 605-612, 2014.

ROLIM, S. G.; COUTO, H. T. Z.; JESUS, R. M.; FRANÇA, J. T. Modelos volumétricos para a Floresta Nacional do Taipé-Aquirí, Serra dos Carajás. Acta Amazonica, Manaus, v. 36, n. 1, p. 106-114, 2006.

SEGURA, M.; KANNINEN, M. Allometric models for tree volume and total aboveground biomass in a tropical humid forest in Costa Rica. Biotropica, Lawrence, v. 37, n. 1, p. 2-8, 2005.

SOUZA, C. A. M.; FINGER, C. A. G.; SCHNEIDER, P. R.; SILVA, G. F. THOMAS, C. Eficiência de um modelo de afilamento ajustado com e sem estratificação por classe de quociente de forma para a formação dos sortimentos de Pinus taeda L. Ciência Florestal, Santa Maria, v. 22, n. 1, p. 125-135, 2012.

TER STEEGE, H.; PITMAN, N.C.A.; SABATIER, D.; et al. Hyperdominance in the Amazonian Tree Flora. Science, Washington, v. 342, n. 6156, p. 324-334, 2013.

THAINES, F; BRAZ, E. M.; MATTOS, P. P.; THAINES, A. A. R. Equações para a estimativa de volume de madeira para a região da bacia do Rio Ituxi, Lábrea, AM. Pesquisa Florestal Brasileira, Brasília, v. 30, n. 64, p. 283$289,2010$.

TONINI, H.; BORGES, R. A. Equação de volume para espécies comerciais em Floresta Ombrófila Densa no Sul de Roraima. Pesquisa Florestal Brasileira, Brasília, v. 35, n. 82, p. 11-17, 2015.

Recebido em 15/03/2016

Aceito para publicação em 10/01/2017 